\title{
Pathologic Stage IIB Gastroesophageal Junction Adenocarcinoma AJCC v8
}

National Cancer Institute

\section{Source}

National Cancer Institute. Pathologic Stage IIB Gastroesophageal Junction

Adenocarcinoma A/CC v8. NCI Thesaurus. Code C133573.

Stage IIB includes: (T1, N1, M0, Any G); (T3, N0, M0, Any G). T1: T umor invades the lamina propria, muscularis mucosae, or submucosa. T3: Tumor invades adventitia. N0: No regional lymph node metastasis. N1: T umor with metastasis in one or two regional lymph nodes. M0: No distant metastasis. (AJCC 8th ed.) 